\title{
PREVALENCE OF NASH AMONG DIABETES
}

\author{
Sheela Krishnamurthy 1
}

${ }_{1}^{1}$ Assistant Professor, Department of General Medicine, BGS GIMS Hospital, Bangalore, Karnataka, India.

\section{ABSTRACT}

\section{BACKGROUND}

NASH, or non-alcoholic steatohepatitis, was originally coined by Dr. Ludwig nearly 30 years ago to describe a distinctive entity considered by fatty changes with lobular hepatitis in the absence of a history of alcoholism. The main lesions described were steatosis, liver cell injury, and the unique zone 3 "chicken wire" fibrosis that remain central in establishing the diagnosis. Due to very few population-based studies and reliable non-invasive screening tools, accurate epidemiological data is not available in India. The incidence of NASH is initiated by many factors, including both environmental and genetics, therefore difficult to define.

\section{MATERIALS AND METHODS}

Patients with Type 2 Diabetes Mellitus were screened for steatohepatitis by ultrasonography.

\section{RESULTS}

The data showed a strong correlation between NASH, insulin resistance, obesity and dyslipidaemia.

\section{CONCLUSION}

Screening for coronary risk factors is important to the work-up and management of patients with Type 2 Diabetes Mellitus.

\section{KEY WORDS}

NASH, Insulin Resistance, Type 2 Diabetes Mellitus.

HOW TO CITE THIS ARTICLE: Krishnamurthy S. Prevalence of NASH among diabetes. J. Evolution Med. Dent. Sci. 2019;8(07):451453, DOI: $10.14260 /$ jemds/2019/99

\section{BACKGROUND}

Non-alcoholic steatohepatitis (NASH) is a pathological state defined excess deposition of fat in the liver ranging from steatosis to hepatitis, cirrhosis and infrequently hepatocellular carcinoma (HCC) without history of ample alcohol intake.[1] Associated mechanisms of NASH include diabetes mellitus, obesity and dyslipidemia which make a direct correlation with the metabolic syndrome.[2] The rise in incidence of obesity and diabetes is causing NASH to become a major public health problem.[3] The over-all incidence of NASH is 15 to $40 \%$ in western countries and 9-40\% in Asian countries.[4] Especially China and Japan are seeing an increase in the incidence of NASH.[5] The risk factors predisposing for NASH are diabetes mellitus, obesity, and insulin resistance. As in India over the last two decades there is been an increase in the incidence of diabetes, obesity and insulin resistance, so it is expected to also see an increase in the incidence of NASH in India. ${ }^{6,7]}$ However the data on the incidence of NASH in India is deficient. [8,9] Hence this study was intended to estimate the incidence of NASH in a population of patients with Type 2 diabetes mellitus.

The mechanism behind NASH has been proposed that it is a 2-hit hypothesis.[10] The first hit involves an imbalance of fatty acid metabolism leading to hepatic triglyceride accumulation (Steatosis). The second hit is metabolic or stress and unregulated cytokine production as a result of efforts to balance the changed lipid homeostasis.

'Financial or Other Competing Interest': None.

Submission 02-12-2018, Peer Review 30-01-2019,

Acceptance 07-02-2019, Published 18-02-2019.

Corresponding Author:

Dr. Sheela Krishnamurthy,

Assistant Professor, Department of General Medicine,

BGS GIMS Hospital, Bangalore, Karnataka, India.

E-mail:sheelakm@gmail.com

DOI: $10.14260 /$ jemds $/ 2019 / 99$
This leads to inflammation and fibrosis. Studies have shown that hepatic mitochondrial dysfunction is vital to the pathogenesis of NASH. [11]

Nonalcoholic steatohepatitis (NASH) is asymptomatic and often diagnosed incidentally by elevated liver enzyme levels and subsequently abdominal ultrasonograph.[12] NASHrelated cirrhosis is now one of the main indications for liver transplantation in the United States. Few population-based studies and reliable noninvasive screening tools make accurate epidemiological data unavailable in India.[13] There is debate about the methods used to make a diagnosis of $\mathrm{NASH}$, and it is still unclear on the clinical associations of histologic changes. The incidence of NASH is affected by many factors, both environmental and genetics, therefore it is difficult to define.

\section{Objectives}

To estimate the prevalence of non-alcoholic steatohepatitis (NASH) by ultrasonography in patients with Type 2 Diabetes Mellitus.

\section{Methodology}

Patients with type 2 diabetes were recruited. History and physical examination findings were documented.

A cross-sectional study was done to look for the incidence of steatohepatitis in Type 2 Diabetes Mellitus patients. The study was conducted in BGS GIMS Hospital from August 2017 to August 2018. The inclusion criteria were patients with Type 2 Diabetes Mellitus. Exclusion criteria included patients with history of chronic ethanol abuse and pre-existing liver disease due to any other known cause (for example, Wilson's Disease or alpha-1 antitrypsin disorder). The sampling method used was simple random sampling. A sample size of 120 'subjects' is needed to estimate a 95\% CI. Obtaining a confidence interval of $99 \%$ would have be even more 
statistically significant but since the study involves subjecting the patients to a battery of investigations including abdominal ultrasonography, a CI of $95 \%$ was acceptable and valid. The statistical test used in this study was the chi square test as both the descriptive method and the inferential method was performed. Data was compared between two groups of patients, those with NASH and those without. This sample population results can be used to make generalizations about larger populations from which the samples were randomly taken.

Fasting blood glucose, liver function tests, lipid profile and glycated hemoglobin were obtained. A diagnosis of NASH was determined on the basis of ultrasound assessment of the liver.

\section{MATERIALS AND METHODS Study Design}

This was a Cross-sectional study.

\section{Study Place}

Study was planned and executed by the Department of General Medicine, BGS Global institute of medical sciences and Hospital, Bangalore, Karnataka during August 2017 to August 2018.

\section{Study Population}

The inclusion criteria were patients with Type 2 Diabetes Mellitus. Exclusion criteria included patients with history of chronic ethanol abuse and pre-existing liver disease due to any other known cause (For example, Wilson's Disease or alpha-1 antitrypsin disorder)

\section{Sampling Technique}

We have used Universal sampling technique all the patients with the inclusion and exclusion criteria.

\section{Sample Size}

The sample size was calculated using standard methods based on the results of previous studies.

Sample size formula,

$n=\left[\frac{Z^{2} * P *(1-P)}{d^{2}}\right]$

\section{Where,}

$\mathrm{n}=$ Sample size,

$\mathrm{Z}=$ Standard normal deviate, (1.96 for $95 \%$ confidence level),

$\mathrm{P}=$ prevalence of NASH in Bangalore (0.31).

Then estimated sample size was $n=128$ (We have collected more than 128 samples i.e. 129) Hence $n=129$

\section{Data Analysis \& Statistics}

The data were entered in Microsoft Excel 2010 and analysed using SPSS software V 20.

Descriptive statistics was used, and results were expressed as percentage and Z-test for two sample means and proportions was applied to check for the significant difference with various factors between NASH \& Non-NASH.
RESULTS

\begin{tabular}{|c|c|c|c|c|c|c|}
\hline \multirow{2}{*}{ Variables } & $\begin{array}{c}\text { NASH } \\
(\mathbf{n = 7 4})\end{array}$ & $\begin{array}{c}\text { Non-NASH } \\
(\mathbf{n = 5 5})\end{array}$ & $\begin{array}{c}\text { Statist } \\
\text { ic }\end{array}$ & p-value \\
\cline { 2 - 6 } & Mean & SD & Mean & SD & Z-test & \\
\hline FBS (mg \%) & 146 & 22 & 144.2 & 18 & 0.507 & 0.6122 \\
\hline $\begin{array}{c}\text { Total chol } \\
\text { (mg \%) }\end{array}$ & 184 & 46 & 179 & 36 & 0.690 & 0.4902 \\
\hline HDL (mg \%) & 35.2 & 8.7 & 39.6 & 13 & 2.225 & 0.0261 \\
\hline LDL (mg \%) & 99.7 & 29 & 92.9 & 45 & 0.989 & 0.3227 \\
\hline Trigly (mg \%) & 176.5 & 43 & 159.6 & 32 & 2.587 & 0.0097 \\
\hline AST (IU/L) & 22.3 & 8.3 & 17.7 & 5.9 & 3.678 & 0.0002 \\
\hline ALT (IU/L) & 25.5 & 4.8 & 19.4 & 7.6 & 5.228 & 0.00001 \\
\hline Table 1. Comparison of mean blood investigations \\
between the two subgroups \\
\hline \multicolumn{7}{|c|}{ p < 0.05 are Significant } \\
\hline \multicolumn{7}{|c|}{ p 0.01 highly significant } \\
\hline
\end{tabular}

\begin{tabular}{|c|c|c|c|c|c|c|}
\hline \multirow{2}{*}{ Variables } & \multicolumn{2}{|c|}{$\begin{array}{c}\text { NASH } \\
(\mathbf{n = 7 4 )}\end{array}$} & $\begin{array}{c}\text { Non-NASH } \\
(\mathbf{n = 5 5 )}\end{array}$ & Statistic & \multirow{2}{*}{ p-value } \\
\cline { 2 - 6 } & $\mathbf{N}$ & $\mathbf{\%}$ & $\mathbf{N}$ & $\mathbf{\%}$ & Z-Test & \\
\hline $\begin{array}{c}\text { Systemic } \\
\text { Hypertension }\end{array}$ & 49 & 66.2 & 30 & 54.5 & 1.345 & 0.1786 \\
\hline Smoking & 12 & 16.2 & 3 & 5.5 & 2.043 & 0.0411 \\
\hline BMI > 25 & 55 & 74.3 & 23 & 41.8 & 3.885 & 0.0001 \\
\hline HbA1c $>7$ & 50 & 67.6 & 26 & 47.3 & 2.345 & 0.0190 \\
\hline \multicolumn{7}{|c|}{ Table 2. Risk Factors for CAD } \\
\hline \multicolumn{7}{|c|}{ p < 0.05 are Significant } \\
\hline
\end{tabular}

Type 2 diabetic patients $(n=129)$ were separated into a NASH group $(n=74)$ and a non-NASH group $(n=55)$. The incidence of NASH was $65 \%$.

A total of 129 patients with Type 2 diabetes mellitus (59 women and 70 men) were studied. The incidence of NASH was $65 \%$, with men having a higher incidence (56.3\%) as compared to women $(43.7 \%)$. Fatty liver showed a bimodal peak with a higher proportion in males.

An $\mathrm{HbA1c}>7 \%$ was used as a measure for uncontrolled blood glucose levels. $85 \%$ in the NASH subgroup and $89 \%$ in the non-NASH subgroup had poor glycaemic control (Odds ratio=0.87). As defined by IDF (2005) criteria, metabolic syndrome was present in $65 \%$ of the study group.

\section{DISCUSSION}

Type 2 diabetes mellitus, obesity, dyslipidaemia, and hypertension contribute to the risk for liver disease and the disease progression. The presence of multiple metabolic abnormalities is associated with the severity of liver disease. The incidence of the metabolic syndrome was considerably higher in the NASH group, as compared to the non-NASH group (61.9\% vs. 13.2\%). Mean liver enzymes (AST and ALT) were higher in the NASH group. More hepatic steatosis was associated with more deviations in the lipid profile and less glycaemic control. Nonetheless, the metabolic syndrome was equally present in both subgroups. Liver enzymes were in higher grades in the patients with NASH $(p=0.156)$. A.K Agarwal et al found that NASH $(p=0.016)$, LDL cholesterol $(\mathrm{p}=0.049)$, hypertension $(\mathrm{p}=0.013)$ and microalbuminuria $(\mathrm{p}$ $=0.034$ ) were independent predictors of CAD. In this study, the incidence of NASH identified by ultrasonography, was $65 \%$. 
A shortcoming of this study is that a diagnosis of NASH was established by ultrasonography and a confirmation test by liver biopsy was not done. However, the most practical technique of identifying NASH in a clinical setting is by ultrasonography and it has a very good sensitivity and specificity. The sensitivity and specificity of ultrasound for identifying hepatic steatosis varies from 60 to $94 \%$ and 88 to $95 \%$, respectively.

\section{Studies suggest that Liver Biopsy is Seldom Necessary to Diagnose NASH}

- Cut-off for fasting plasma glucose has been lowered to $100 \mathrm{mg} / \mathrm{dl}$.

- Central obesity is now a mandatory variable

- The cut-offs of waist circumference have been lowered (male, $94 \mathrm{~cm}$; female, $80 \mathrm{~cm}$ ), and for south Asians: (Male, $90 \mathrm{~cm}$; female, $80 \mathrm{~cm}$ )

Is NASH a novel component of the metabolic syndrome and a risk factor for coronary artery disease? A convincing number of studies have correlated NASH with cardiovascular disease. Due to limitations, including study design and method of diagnosing, it makes it difficult to reach an absolute inference. Prospective studies show a substantial increase in unfavourable outcomes of patients with NASH compared to the general population. These studies divulge that coronary artery disease is the leading or no less than the second leading cause of death. Nevertheless, these patients also had coincidental components of the metabolic syndrome, including insulin resistance, dyslipidaemia, obesity and hypertension.

\section{CONCLUSION}

Screening for NASH and other coronary risk factors is important in the work-up and management of patients with Type 2 Diabetes Mellitus. Is NASH a novel component of the metabolic syndrome and a risk factor for coronary artery disease? A convincing number of studies have correlated NASH with cardiovascular disease. Due to limitations, including study design and method of diagnosing, it makes it difficult to reach an absolute inference. Prospective studies show a substantial increase in unfavourable outcomes of patients with NASH compared to the general population. These studies divulge that coronary artery disease is the leading or no less than the second leading cause of death. Nevertheless, these patients also had coincidental components of the metabolic syndrome, including insulin resistance, dyslipidaemia, obesity and hypertension.

\section{REFERENCES}

[1] Farrell GC, Larter CZ. Nonalcoholic fatty liver disease: from steatosis to cirrhosis. Hepatology 2006;43(2 Suppl 1):S99-S112.

[2] McCullough AJ. Thiazolidinediones for nonalcoholic steatohepatitis-promising but not ready for prime time. N Engl J Med 2006;355(22):2361-3.

[3] Yoon KH, Lee JH, Kim JW, et al. Epidemic obesity and type 2 diabetes in Asia. Lancet 2006;368(9548):16818.

[4] Clark JM. Epidemiology of nonalcoholic fatty liver disease in adults. J Clin Gastroenterol 2006;40(Suppl 1):S5-S10.

[5] Amarapurkar DN, Hashimoto E, Lesmana LA, et al. How common is NAFLD in the Asia-Pacific Region and are there local differences? J Gastroenterol Hepatol 2007;22(6):788-93.

[6] Mohan V, Deepa R. Adipocytokines and the expanding 'Asian Indian Phenotype'. J Assoc Physic India 2006;54:685-6.

[7] Misra A, Vikram NK. Insulin resistance syndrome (metabolic syndrome) and Asian Indian. Current Science 2002;83:1483-96.

[8] Singh SP, Nayak S, Swain M, et al. Prevalence of nonalcoholic fatty liver disease in coastal eastern India; a preliminary ultrasonographic survey. Trop Gastroenterol 2004;25(2):76-9.

[9] Duseja A, Chawla Y. Nonalcoholic fatty liver disease in India How much? How Soon? Trop Gastroenterol 2005;26(1):1-3.

[10] Ludwig J, Viggiano TR, McGill DB, et al. Nonalcoholic steatohepatitis: Mayo Clinic experiences with a hitherto unnamed disease. Mayo Clin Proc 1980;55(7):434-8.

[11] Chitturi S, Farrell GC, Hashimoto E, et al. Non-alcoholic fatty liver disease in the Asia-Pacific region: definitions and overview of proposed guidelines. J Gastroenterol Hepatol 2007;22(6):778-87.

[12] Browning JD, Szczepaniak LS, Dobbins R, et al. Prevalence of hepatic steatosis in an urban population in the United States: impact of ethnicity. Hepatology 2004;40(6):1387-95.

[13] Weston SR, Leyden W, Murphy R, et al. Racial and ethnic distribution of nonalcoholic fatty liver in persons with newly diagnosed chronic liver disease. Hepatology 2005;41(2):372-9. 